\title{
Tecnura
}

\section{Ajuste de la protección de sobreexcitación ANSI 24 para un generador sincrónico en un relé SIEMENS 7UM62}

\section{Adjusting ANSI 24 overexcitation protection in a synchronous generator for a SIEMENS 7UM62 relay}

\author{
Nayiver Gladys Caicedo Delgado¹, Carlos Alberto Andrade Alegría ${ }^{2}$
}

Fecha de recepción: 27 de agosto de 2018

Fecha de aceptación: 15 de febrero de 2019

Cómo citar: Caicedo D., G. y Andrade, C.A. (2019). Ajuste de la protección de sobreexcitación ANSI 24 para un generador sincrónico en un relé SIEMENS 7UM62. Tecnura, 23(61), 57-68. doi: https://doi. org/10.14483/22487638.13785

\section{Resumen}

Contexto: Las funciones de protección de un grupo generador-transformador deben garantizar su vida útil cuando se presenta una condición de operación anormal, de esta manera se mejora la confiabilidad del servicio de energía eléctrica en el sistema eléctrico de potencia. Cuando la relación V/Hz del grupo se incrementa puede presentarse saturación en el núcleo magnético, induciendo un flujo de dispersión en los componentes no laminados que no están diseñados para conducirlo y puede ocurrir un daño en segundos; razón por la cual, estas máquinas se protegen con la función de protección (FDP) ANSI 24.

Metodología: Este artículo presenta la teoría que justifica la implementación de la FDP ANSI 24 en un grupo generador-transformador y la metodología para calcular cada uno de sus parámetros de ajuste en un relé multifuncional Siemens 7UM62. La originalidad del artículo radica en que se explica su diagrama lógico de decisión de una manera clara y detallada, identificando las señales de entrada: ajustes, analógicas y binarias, y sus señales de salida.
Este diagrama lógico se organizó en 3 subdiagramas para facilitar su comprensión e identificar las causas que originan sus salidas de alarmas, arranque, disparos o bloqueo.

Resultados: Se obtuvo el diagrama de decisión detaIlado de la FDP ANSI 24, se describieron los criterios de ajuste de entrada de cada uno de los parámetros de ajustes y un procedimiento detallado para calcular cada uno. También, se obtuvieron los resultados numéricos de todos los ajustes para el grupo generador-transformador de la Unidad 1 de la Central Hidroeléctrica de Salvajina.

Conclusiones: Se recomienda para una mayor precisión en los parámetros de ajuste obtener la característica de calentamiento $\mathrm{V} / \mathrm{Hz}$ real o entregada por el fabricante de los equipos a proteger.

Palabras claves: ANSI 24, generador sincrónico, protecciones eléctricas.

\section{Abstract}

Context: The protection functions of a generatortransformer group must guarantee its useful life when an abnormal operating condition arises, thus

1 Ingeniera electricista, magíster en Sistemas de Generación de Energía, doctora en Ingeniería. Línea de énfasis en Ingeniería Eléctrica y Electrónica de la Universidad del Valle. Profesora titular de la Escuela de Ingeniería Eléctrica y Electrónica de la Universidad del Valle. Cali, Colombia. Contacto: nayiver.gladys.caicedo@correounivalle.edu.co ORCID: https://orcid.org/0000-0002-8679-7465

2 Ingeniero electricista, Ingeniero electricista de la Empresa Proing S.A. Su línea de trabajo es el diseño eléctrico en Cali. Cali, Colombia. Contacto:auxiliardiseno2@proing.com ORCID: https://orcid.org/0000-0002-7698-7644 
improving the reliability of the electric power service in the electric power system. When the $\mathrm{V} / \mathrm{Hz}$ ratio of the group increases, saturation may occur in the magnetic core and induce a dispersion flow in the non-laminated components that are not designed to conduct it, and damage can occur in seconds. This is the reason why machines are protected with the ANSI 24 protection function (FDP).

Methodology: This article presents the theory that justifies the implementation of the ANSI 24 protection function in a generator-transformer group, as well as the methodology to calculate each of the adjustment parameters in a SIEMENS 7UM62 multifunctional relay. The article explains the logical decision diagram in a clear and detailed way, identifying the settings, input signals (analog and binary), and output signals. The logical diagram was organized in three subdiagrams to facilitate understanding and to identify the causes that originate its alarm, start, trip, or block outputs.

Results: The detailed decision diagram of the ANSI 24 protection function was obtained; the input adjustment criteria of the adjustment parameters and a detailed procedure for calculating each were described. In addition, the numerical results of all the adjustments for the generator-transformer group of Unit 1 of the Salvajina hydroelectric power station were obtained.

Conclusions: The results of this research recommend obtaining the actual $\mathrm{V} / \mathrm{Hz}$ heating characteristic or confirming the one delivered by the manufacturer of the equipment to be protected, so that it is possible to have greater precision in the adjustment parameters.

Keywords: ANSI 24, synchronous generator, electrical protections

\section{INTRODUCCIÓN}

Los generadores sincrónicos son los encargados de suministrar potencia eléctrica a las diferentes cargas, garantizando un proceso continuo y efectivo durante su funcionamiento. Razón por lo cual se han implementado diferentes funciones de protección que permiten proteger la máquina al presentarse distintas fallas propias del generador o del sistema eléctrico de potencia al que está conectado (Muñoz y Caicedo, 2018).

Cuando hay desviaciones en frecuencia o tensión, se pueden presentar esfuerzos térmicos y dieléctricos que pueden causar grandes daños de manera rápida en el generador o transformador. Por ello, se debe garantizar que se cumplan las normas de los límites operativos especificados por sus fabricantes.

La sobreexcitación medida en bornes del generador se da cuando la magnitud que relaciona la tensión y la frecuencia expresada como voltios/ Hertz $(\mathrm{V} / \mathrm{Hz})$, sobrepasa los límites establecidos por las normas ANSI/IEEE definidas en IEEE Guide for AC Generator Protection (2006), la cual para los generadores es 1,05 p.u. (base del generador) y para los transformadores es 1,05 p.u. (base del secundario del transformador) a carga nominal, y f.p. de 0,8 o 1,1 p.u. (base del transformador) sin carga.

Cuando la relación de $\mathrm{V} / \mathrm{Hz}$ es excedida de un valor puede haber saturación en el núcleo magnético del generador o transformador, induciendo un flujo de dispersión en componentes no laminados que no están diseñados para soportarlo, y se producen pérdidas por corrientes parásitas en los componentes adyacentes al núcleo, como en cuñas de ranura o componentes de ensamblaje; este flujo puede producir un daño en segundos. Estas pérdidas producen un incremento de la temperatura del equipo, lo que ocasiona un deterioro del aislamiento en segundos; por esta razón, las máquinas se protegen con la FDP ANSI 24. Esta calcula en porciento o en unidad el nivel de sobreexcitación en la máquina; es decir, la relación entre la tensión y la frecuencia $(\mathrm{V} / \mathrm{Hz})$. A mayor porcentaje mayor sobreexcitación y a menor porcentaje menor sobreexcitación. Las curvas límite de sobreexcitación (V/Hz vs. tiempo) de los generadores y 
transformadores en operación sin carga deben ser suministradas por sus respectivos fabricantes.

En la figura 1 se ilustran las dos etapas básicas de protección de la función $\mathrm{V} / \mathrm{Hz}$ : la de tiempo definido y la de tiempo inverso. Las regiones sombreadas corresponden a las zonas protegidas por las etapas de protección.

Para un grupo generador-transformador se estandarizan sus dos curvas, es decir se deben referir a una misma tensión base, que generalmente es a la tensión nominal de salida del generador.
En la figura 2 se presentan tres esquemas de protección comúnmente empleados en la industria para esta función de protección $\mathrm{V} / \mathrm{Hz}$. Una desventaja de emplear un esquema de protección que solo utilice dos características de tiempo definido consiste en que pueden quedar áreas del grupo generador-transformador sin proteger. Por esta razón, se combinan las funciones de protección de tiempo definido con las de tiempo inverso, ya que proporcionan una mejor coordinación con los límites operativos del equipo.

En la figura 3 se muestra el resultado de unificar las curvas para un grupo generador transformador.
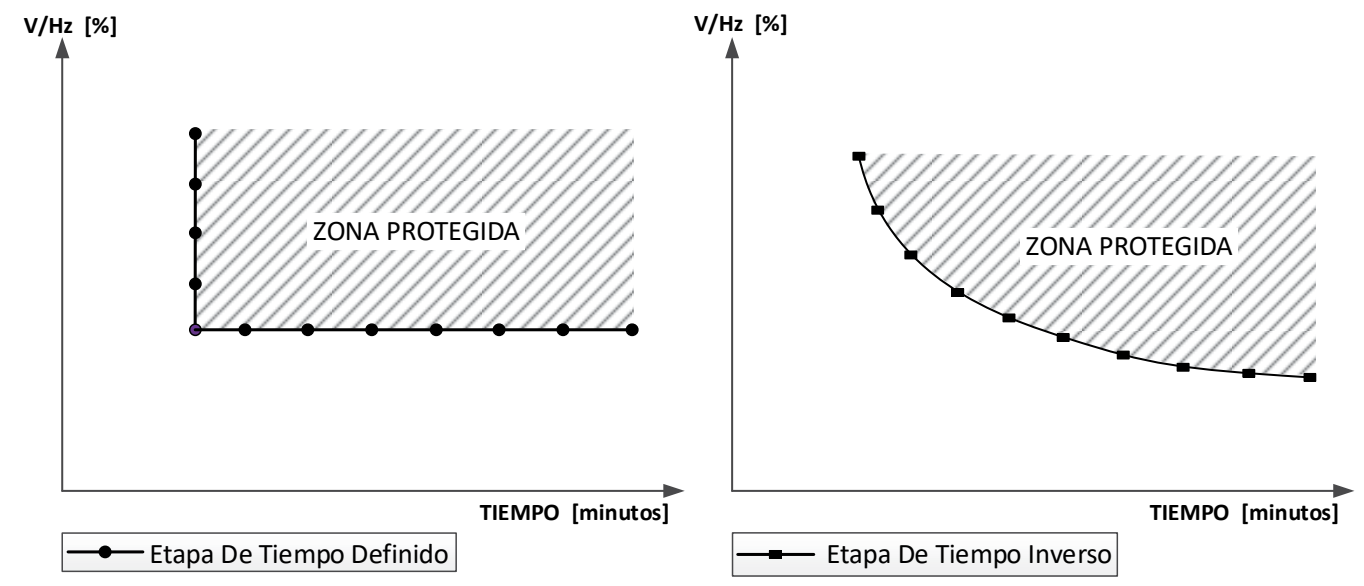

Figura 1. Curvas límite $\mathrm{V} / \mathrm{Hz}$ sin carga de generadores y transformadores

Fuente: Siemens (2009).

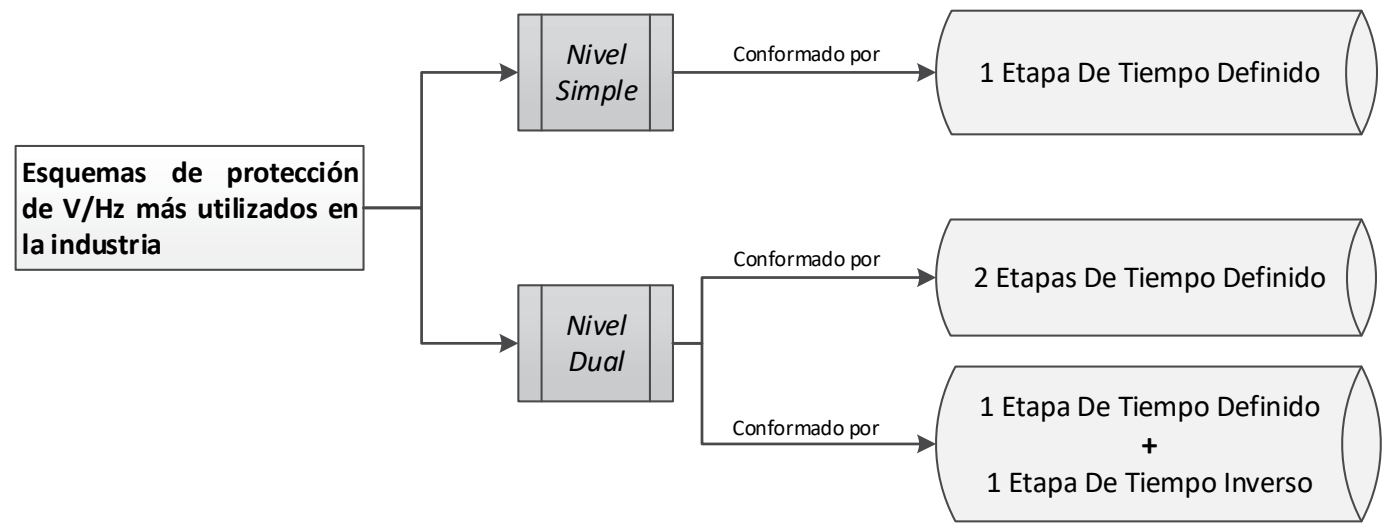

Figura 2. Esquemas $\mathrm{V} / \mathrm{Hz}$ utilizados en un grupo generador-transformador

Fuente: Ceballos (2014). 


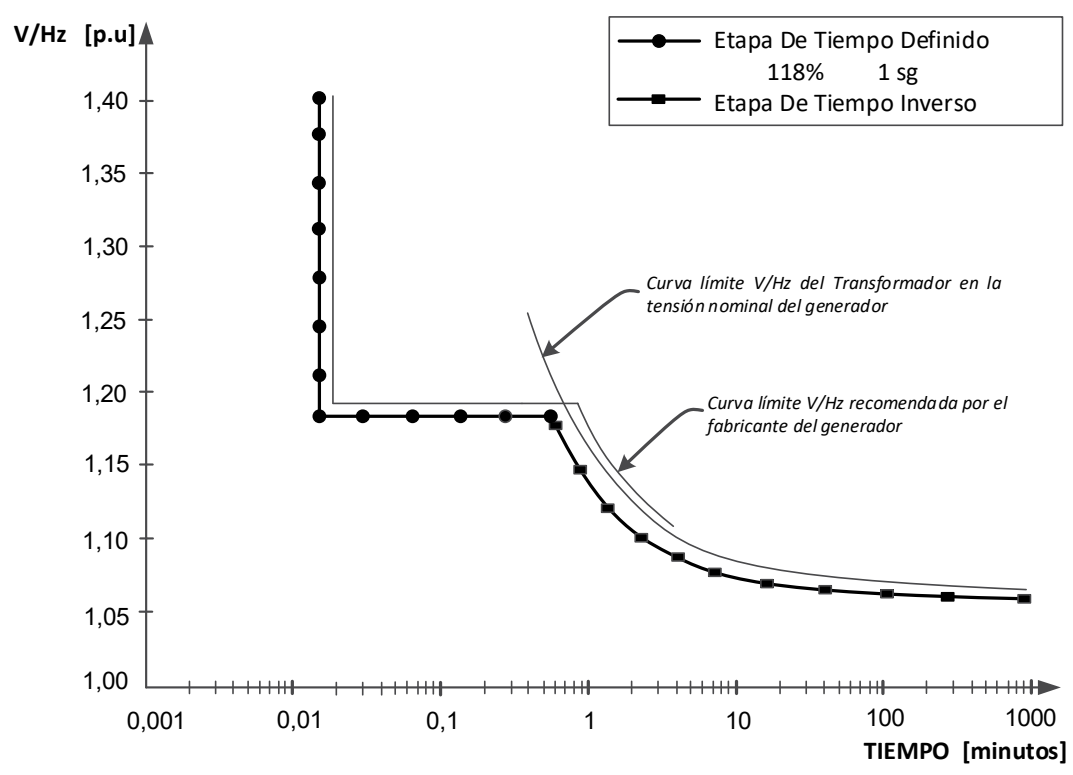

Figura 3. Curva limite $\mathrm{V} / \mathrm{Hz}$ de un grupo generador-transformador para una etapa de tiempo definido más otra de tiempo inverso

Fuente: Ceballos (2014).

\section{¿Qué causa la sobreexcitación en generadores?}

Una sobreexcitación excesiva en un generador excederá la capacidad del aislamiento del devanado del estator del generador, sin embargo, esta FDP $\mathrm{V} / \mathrm{Hz}$ no podrá detectar todas las condiciones de sobretensión y debe ser respaldada por otras funciones de protección que protejan la máquina en caso de falla.

Según Ceballos (2014), una condición de sobreexcitación puede ocurrir por varias circunstancias:

- Pérdida de una unidad de generación cercana, lo cual afecta el flujo de reactivos y se refleja como una caída de tensión. En un intento de mantener la tensión del sistema mientras que el generador disparado se reconecta, los sistemas de excitación de los generadores restantes tratan de reforzar la tensión terminal, llevando al límite el control de excitación. Si durante este intervalo ocurre una falla en el control de la excitación, habrá una sobreexcitación.
- Un generador operando a niveles nominales puede suministrar al sistema de potencia un nivel alto de VAR y su tensión puede permanecer cerca de los niveles nominales de la red debido a las interconexiones. Si en estas condiciones hay una pérdida súbita de carga o de las interconexiones y los controles de excitación del generador no responden adecuadamente, la tensión de la unidad puede elevarse súbitamente, originando una sobreexcitación.

- Cuando una unidad de generación está conectada al sistema de potencia, a través de líneas de transmisión largas y ocurre una apertura de su interruptor principal, puede darse una autoexcitación, si la admitancia de carga en los terminales del generador es mayor que la admitancia de eje en cuadratura 1/Xq; esto debido a que el regulador de tensión presenta una retroalimentación positiva que puede causar una rápida elevación de tensión (IEEE Guide for AC Generator Protection, 2006).

La figura 4 ilustra una sección transversal axial de un turbogenerador que muestra la densidad del 
campo magnético principal y la densidad del flujo de dispersión. La densidad del campo magnético de dispersión es dañina en los extremos del núcleo magnético del generador, debido a que puede inducir altas corrientes parásitas y por tanto alta temperatura y mayores pérdidas.

\section{Conexión de los TP función ANSI 24}

La función ANSI 24 del relé Siemens 7UM62 mide las tensiones de línea del generador a través de los transformadores de potencial de protección (TP), como se ilustra en la figura 5.

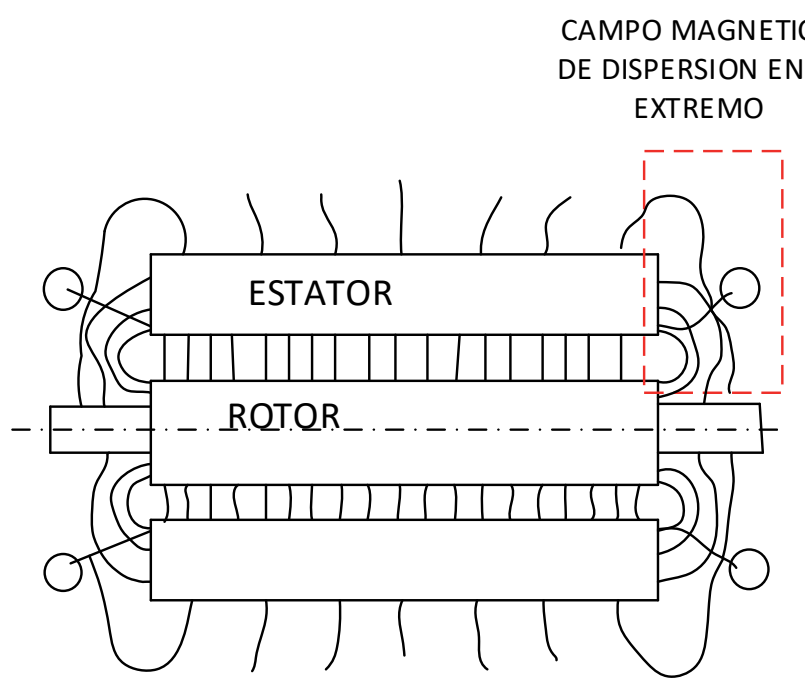

Figura 4. Flujos magnéticos de dispersión en un turbogenerador

Fuente: IEEE Guide for AC Generator Protection (2006).

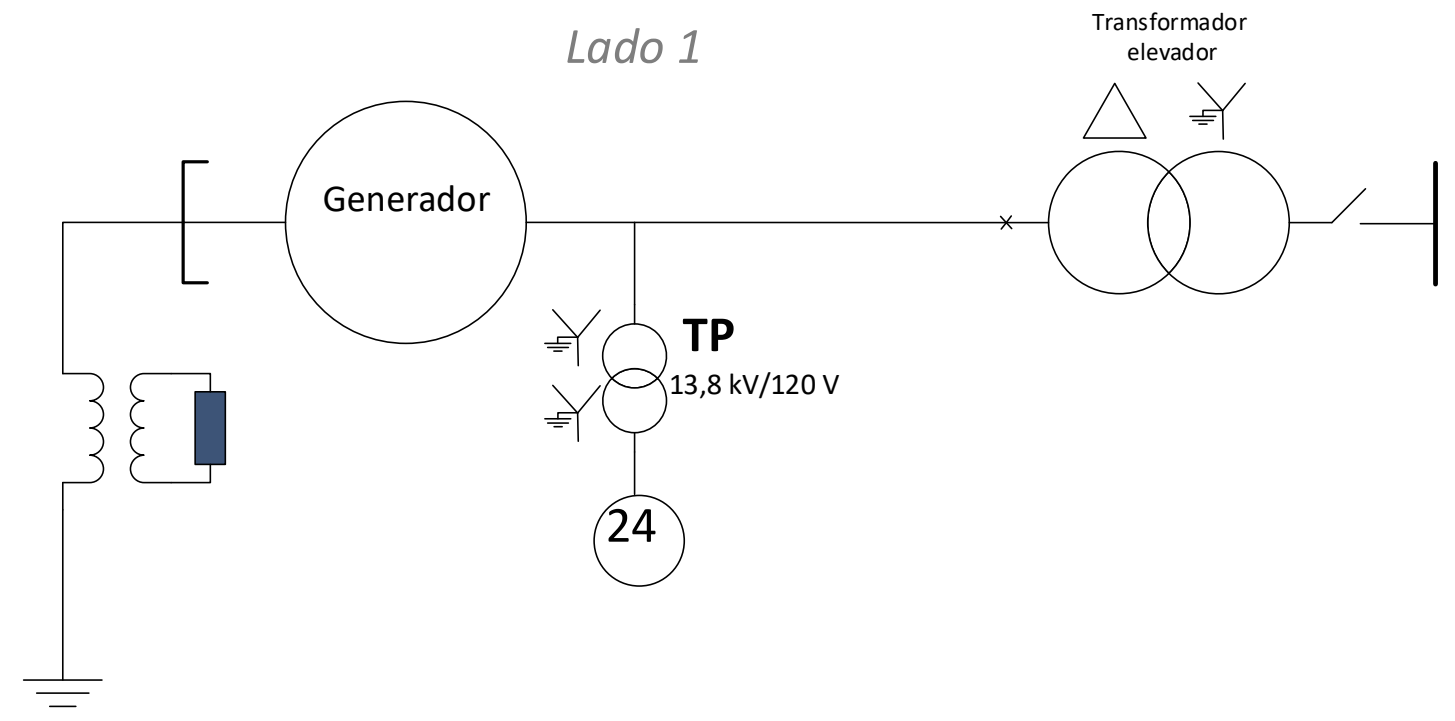

Figura 5. Conexión de los TP en los bornes de un generador para la conexión de la FDP ANSI 24

Fuente: elaboración propia. 
A partir de la señal de tensión, el relé mide la amplitud o el valor en RMS y calcula la frecuencia a partir de esta señal. El nivel de sobreexcitación de manera porcentual o en PU se calcula mediante la ecuación (1).

$$
\text { sobreexcitación }=\frac{\frac{V_{\text {medida }}}{V_{\text {nominal }}}}{\frac{f_{\text {medida }}}{f_{\text {nominal }}}}=\frac{V}{f}[\text { pu o \% }]
$$

La señal de tensión para el relé se toma de una fase del generador, si existe un fusible fundido o una conexión incompleta del circuito y no existe ninguna tensión censada por el relé, no habría protección. Por este motivo, se debe utilizar un TP en cada fase para tener una mejor confiabilidad.

\section{METODOLOGÍA DE AJUSTE DE LA FUNCIÓN ANSI 24}

A continuación, en la metodología para el ajuste de la FDP ANSI 24 se describen: las señales de entrada y salida, los diagramas funcionales de decisión y los criterios de ajuste de las señales de entrada requeridas para el relé Siemens 7UM62.

En la tabla 1 se detallan la dirección cada una de las entradas y su descripción utilizadas en el diagrama lógico de decisión de la función ANSI 24 (SIEMENS, 2009).

Tabla 1. Entradas del diagrama lógico de decisión de la función de protección ANSI 24

\begin{tabular}{|c|c|c|}
\hline Dirección & Entrada & Descripción \\
\hline $\begin{array}{l}\text { Entrada de ajuste } \\
\text { [4301] }\end{array}$ & $\begin{array}{l}\text { (On-Off-Block Relay) Ove- } \\
\text { rexcitation Protection }\end{array}$ & $\begin{array}{l}\text { Para activar la protección } 24 \text { seleccione }(\text { On), para desactivarla selec- } \\
\text { cione }(\text { Off), para bloquear seleccione (Block Relay). }\end{array}$ \\
\hline $\begin{array}{l}\text { Entrada de ajuste } \\
\text { [4302] }\end{array}$ & 24-1 V/f Pickup> & $\begin{array}{l}\text { Primer ajuste de sobreexcitación. Una vez superado este umbral de so- } \\
\text { breexcitación, se habilita la característica de tiempo inverso. También, } \\
\text { se activa una alarma para avisar con un tiempo de retardo. }\end{array}$ \\
\hline $\begin{array}{l}\text { Entrada de ajuste } \\
\text { [4303] }\end{array}$ & 24-1 V/f Time Delay> & $\begin{array}{l}\text { Tiempo de ajuste, el cual inicia una vez superado el primer ajuste de V/ } \\
\mathrm{Hz} \text { (4302). Cuando se termina el tiempo de retardo, se activa la alarma } \\
\text { para dar aviso a los operarios sobre la condición de sobreexcitación. }\end{array}$ \\
\hline $\begin{array}{l}\text { Entrada de ajuste } \\
\text { [4304] }\end{array}$ & 24-2 V/f Pickup >> & $\begin{array}{l}\text { Segundo tiempo de ajuste de sobreexcitación. Este umbral se configura } \\
\text { normalmente en valores altos de } \mathrm{V} / \mathrm{Hz} \text {. Una vez superado este umbral } \\
\text { de sobreexcitación, se emite disparo con un tiempo de retardo. }\end{array}$ \\
\hline $\begin{array}{l}\text { Entrada de ajuste } \\
\text { [4305] }\end{array}$ & 24-2 V/f Time delay>> & $\begin{array}{l}\text { Segundo ajuste de tiempo asociado al segundo ajuste de V/Hz>> } \\
\text { (4304). }\end{array}$ \\
\hline $\begin{array}{l}\text { Entrada de ajuste } \\
{[4306-4313]}\end{array}$ & Time Delay & $\begin{array}{l}\text { Ocho ajustes de tiempo de retardo asociados a los ocho valores de V/ } \\
\text { Hz que conforman la característica de tiempo inverso. }\end{array}$ \\
\hline $\begin{array}{l}\text { Entrada de ajuste } \\
\text { [4314] }\end{array}$ & Time for Cooling Down & $\begin{array}{l}\text { Es el tiempo de enfriamiento del generador hasta su valor de tempera- } \\
\text { tura inicial, después de haber disparado por sobreexcitación. }\end{array}$ \\
\hline
\end{tabular}

Fuente: Siemens (2009).

En la tabla 2 se presentan la dirección de salida y la descripción de las salidas: arranque, disparo y bloqueo de FDP ANSI 24 SIEMENS (2009).

\section{Diagrama lógico de la protección de sobreexcitación (ANSI 24)}

El diagrama lógico de la función de protección ANSI 24 del relé Siemens 7UM62 se compone de tres etapas como se ilustra en (figura 6). La primera, de tiempo definido, calcula la relación $\mathrm{V} /$ $\mathrm{Hz}>$; en esta se define el primer pickup de la sobreexcitación. En la segunda, correspondiente a la característica térmica de la máquina, se construye una curva de tiempo inverso a través de los datos de entrada; aquí el relé arranca, emite alarma y, de ser necesario, da orden de disparo al interruptor principal para evitar calentamiento en el generador. Y, en la tercera etapa, de tiempo definido, se detectan fallas que sobrepasan un valor de pickup 
Tabla 2. Salidas del diagrama lógico de decisión de la función de protección ANSI 24

\begin{tabular}{|c|c|c|}
\hline Dirección & Salida & Descripción \\
\hline Salida [5353] & $\begin{array}{l}\text { Bloqueo externo de la función } \\
\text { ANSI } 24\end{array}$ & $\begin{array}{l}\text { Entrada binaria externa que indica si la función de protección ANSI } \\
24 \text { se encuentra bloqueada. }\end{array}$ \\
\hline Salida [5357] & Reset externo de memoria & Entrada binaria externa para restablecer la memoria de la réplica térmica. \\
\hline Salida [5361] & Anuncio Off de la función ANSI 24 & Indica que la función ANSI 24 se encuentra apagada. \\
\hline Salida [5362] & $\begin{array}{l}\text { Anuncio Bloqueo de la función } \\
\text { ANSI } 24\end{array}$ & $\begin{array}{l}\text { Indica si la función de protección ANSI } 24 \text { se encuentra bloqueada } \\
\text { para evitar disparos al interruptor. }\end{array}$ \\
\hline Salida [5363] & Anuncio On de la función ANSI 24 & Indica que la función ANSI 24 se encuentra encendida. \\
\hline Salida [5367] & Alarma V/Hz> & $\begin{array}{l}\text { Alarma que se activa cuando se cumple el tiempo de retardo de esta } \\
\text { etapa (24-1 V/f }>\text { Time Delay), y que muestra la sobreexcitación excesiva. }\end{array}$ \\
\hline Salida [5369] & Reset memoria & Restablecer la memoria de la réplica térmica. \\
\hline Salida $[5370]$ & Arranque por $\mathrm{V} / \mathrm{Hz}>$ & $\begin{array}{l}\text { Señaliza que la función de protección ANSI } 24 \text { arrancó, cuando el } \\
\text { valor de sobreexcitación supere el valor ajustado en la entrada. }\end{array}$ \\
\hline Salida [5371] & Disparo por $\mathrm{V} / \mathrm{Hz}>>$ & $\begin{array}{l}\text { Disparo que se activa cuando se cumple el tiempo de retardo de esta } \\
\text { etapa }(24-2 \mathrm{~V} / \mathrm{f}>>) \text {, y que muestra la sobreexcitación excesiva. }\end{array}$ \\
\hline
\end{tabular}

Salida [5372] Disparo por $\mathrm{V} / \mathrm{Hz}>$ th.

Disparo que se activa cuando se cumple el tiempo de retardo de esta etapa $(24-1 \mathrm{~V} / \mathrm{f}>)$, y que muestra la sobreexcitación excesiva.

Salida [5373] Arranque por V/Hz>>
valor de sobreexcitación supere el valor ajustado en la entrada.

Fuente: SIEMENS (2009).

V/Hz>>ajustado, para posteriormente despejarlas o dar una señal de alarma.

\section{Subdiagramas lógicos de la función de protección ANSI 24 de relé SIEMENS 7UM62}

A continuación, se describen los 3 subdiagramas de la función de sobreexcitación.

\section{Subdiagrama lógico de sobreexcitación por V/ $\mathrm{Hz>}$ de la función de protección ANSI 24}

En la figura 7 se ilustra el primer subdiagrama lógico de la función de protección ANSI 24, donde se compara el ajuste de pickup del nivel de sobreexcitación $\mathrm{V} / \mathrm{Hz}>$ con el valor real medido, si el ajuste es mayor y la función se encuentra activa y no está bloqueada, se presenta una señal de salida de arranque de la FDP ANSI 24, y activa un contador cuyo tiempo es comparado con un tiempo de permanencia ajustado en la entrada $\mathrm{V} / \mathrm{Hz}$ Time Delay> [4303]; cuando el tiempo del contador es mayor que el tiempo ajustado, se genera una alarma porque el valor de sobreexcitación es mayor que $\mathrm{V} / \mathrm{Hz}$ pickup $>$ y permaneció durante un tiempo mayor que V/Hz Time Delay> [4303].

\section{Subdiagrama lógico de la FDP ANSI 24 por sobreexcitación de la característica de tiempo inverso}

En la figura 8 se ilustra el segundo subdiagrama de la función de protección ANSI 24, que corresponde a la característica térmica de tiempo inverso de la máquina, la cual produce una señal de disparo si se cumplen dos condiciones: a) que el valor $\mathrm{V} / \mathrm{Hz}$ medido se encuentre dentro del rango de la curva de tiempo inverso, lo cual lo verifica mediante los valores de $\mathrm{V} / \mathrm{Hz}>$ y $\mathrm{V} / \mathrm{Hz}$, y b) que la maquina se encuentra fría. Una vez cumplidas estas dos condiciones se activa un contador y se calcula el tiempo de disparo mediante la característica de tiempo inverso. El tiempo del contador es comparado con el tiempo calculado y, cuando este es superado, se da orden de disparo en la zona de característica térmica. 


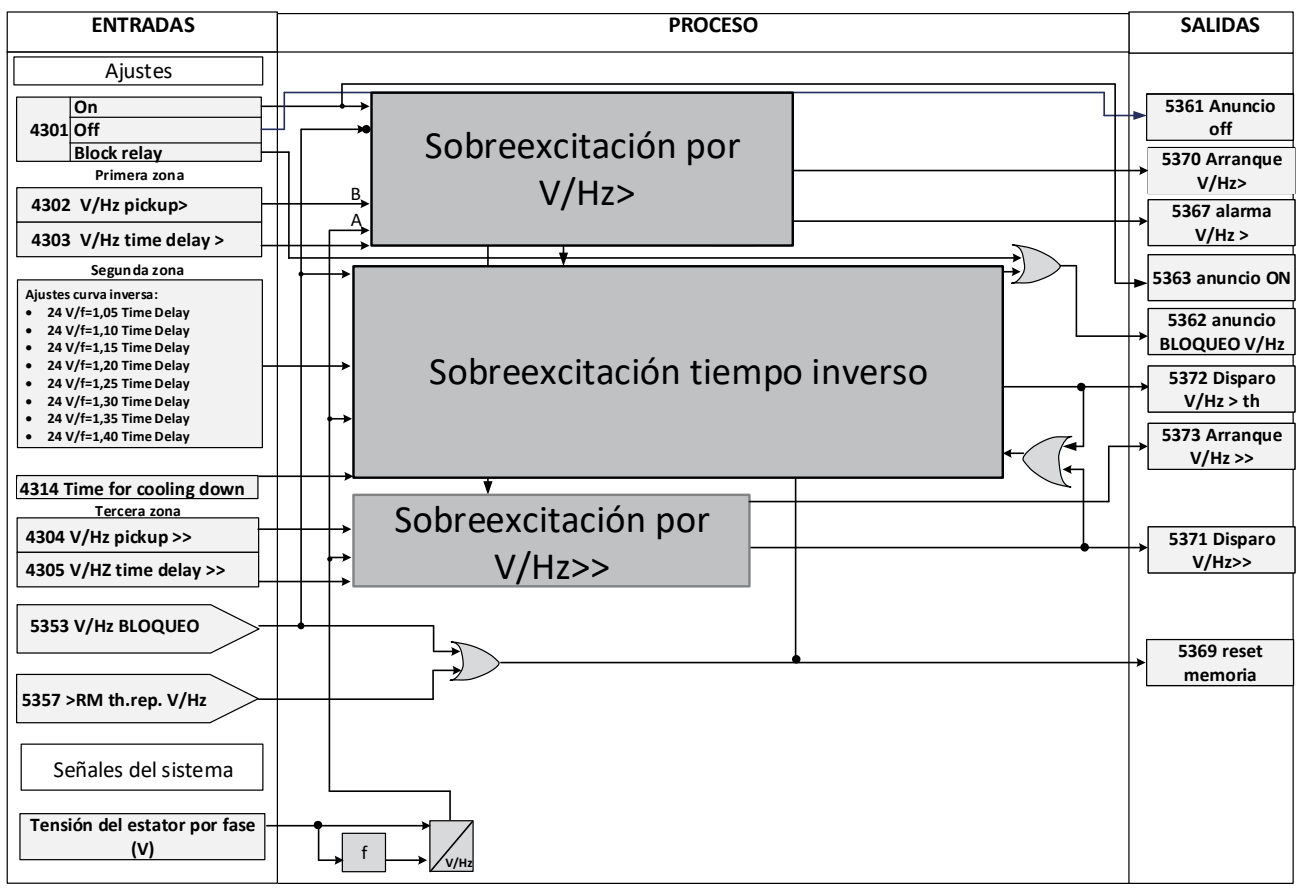

Figura 6. Diagrama lógico de decisión completo de la función ANSI 24

Fuente: elaboración propia.

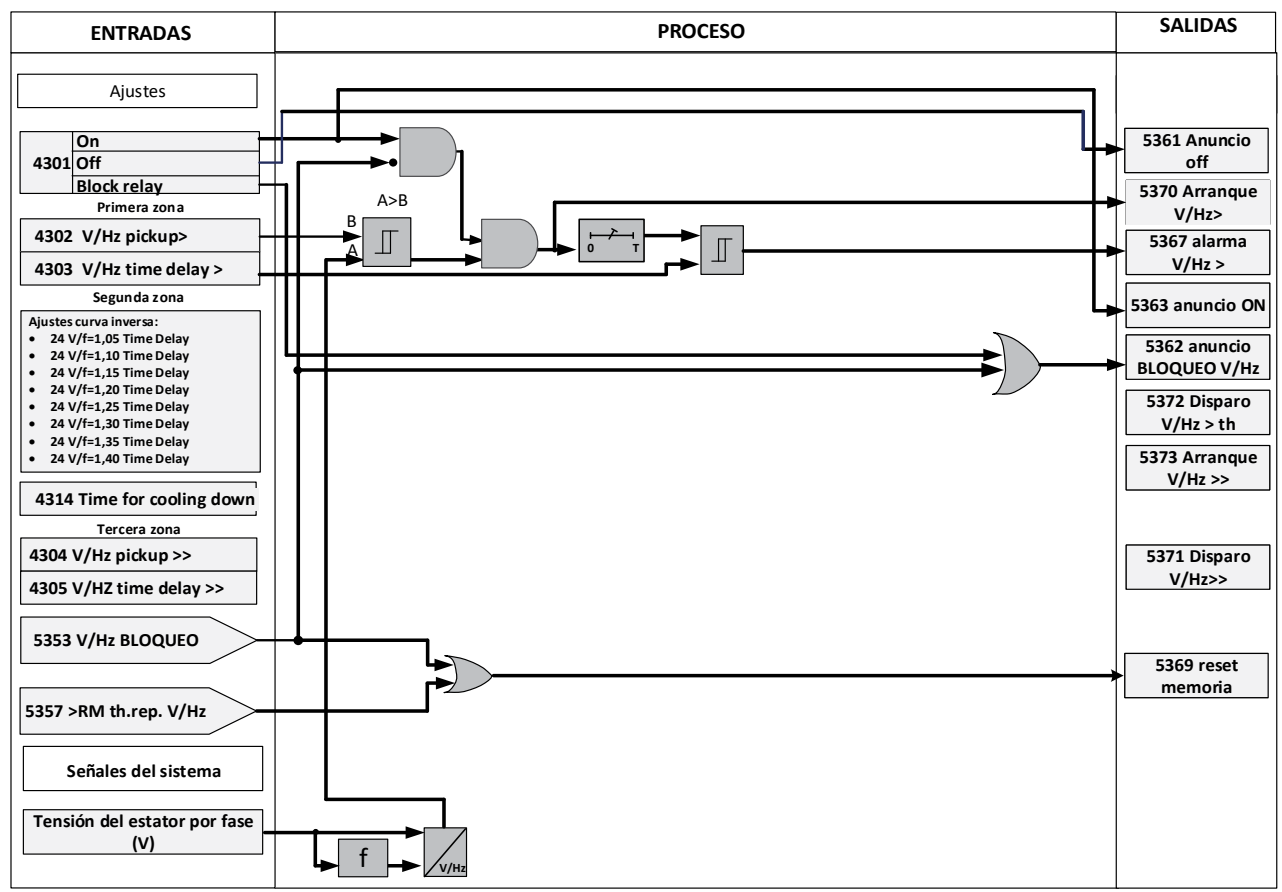

Figura 7. Subdiagrama lógico de decisión de la FDP ANSI 24. Etapa sobreexcitación por V/Hz>

Fuente: elaboración propia. 


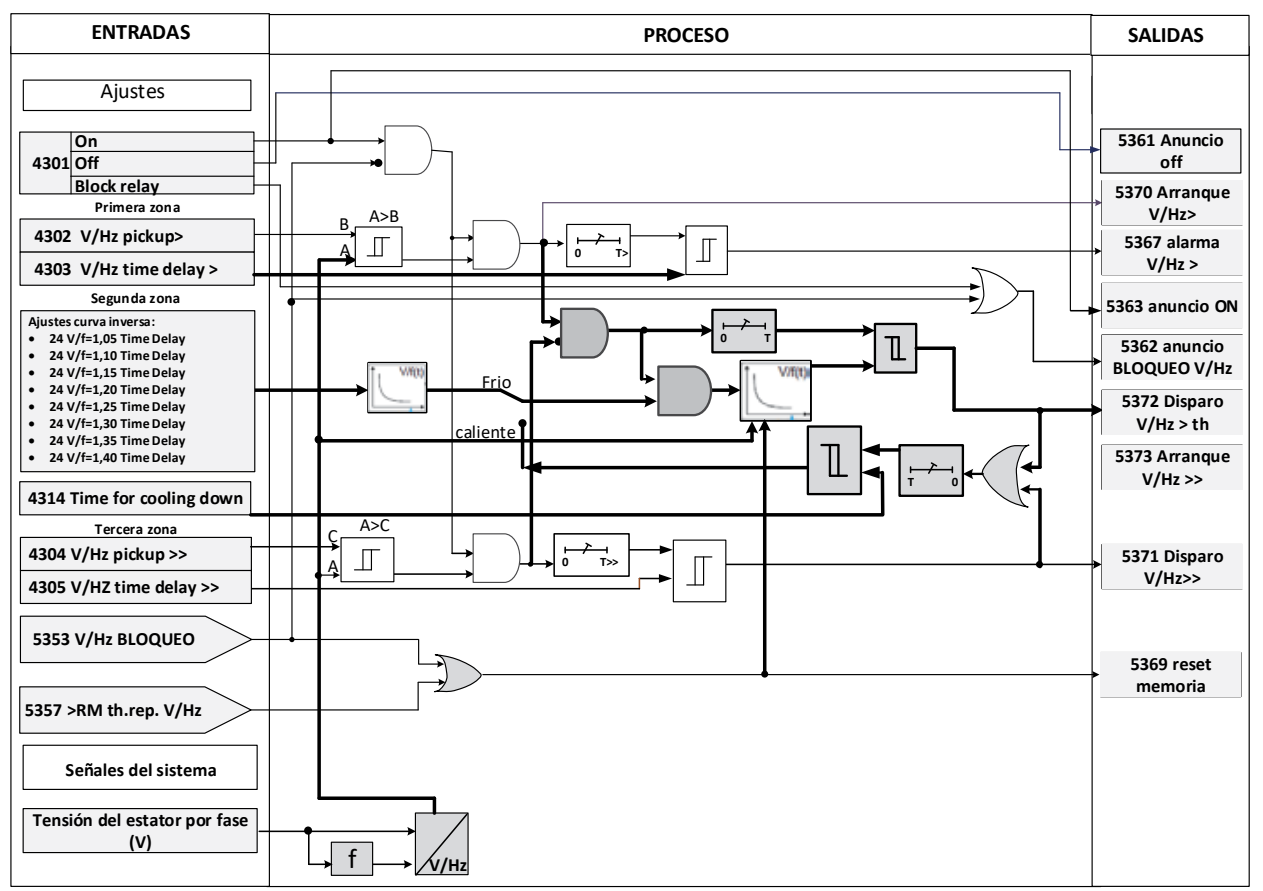

Figura 8. Subdiagrama lógico de decisión de la FDP ANSI 24. Etapa de sobreexcitación por la característica de tiempo inverso

Fuente: elaboración propia.

- Etapa de enfriamiento. Inmediatamente después de la orden de disparo por condición de sobreexcitación en la característica inversa como se ilustra en la Figura 8, se activa un contador cuyo tiempo es comparado con el tiempo de ajuste tfor cooling [4314], mientras el tiempo del contador sea menor al tiempo tfor cooling, la función ANSI 24 permanecerá en estado caliente. Cuando se cumple el tiempo de enfriamiento de la máquina, la función de protección se desbloquea y pasa a estado frío.

\section{Subdiagrama lógico de sobreexcitación por V/} $\mathrm{Hz}>>$ de la función de protección ANSI 24

En la figura 9 se ilustra el tercer subdiagrama lógico de la función de protección ANSI 24, donde se compara el ajuste de pickup del nivel de sobreexcitación $\mathrm{V} / \mathrm{Hz}>>$ con el valor real medido, en este caso los valores de sobreexcitación son muy elevados. Si el ajuste es mayor y la función se encuentra activa y no está bloqueada, se presenta una señal de salida de arranque de la FDP ANSI 24, y activa un contador cuyo tiempo es comparado con un tiempo de permanencia ajustado en la entrada V/Hz Time Delay >> [4305]; cuando se supera el tiempo ajustado se da orden de disparo al interruptor principal asociado al generador.

\section{Ajustes de la función ANSI 24 del relé 7 UM62 para un generador sincrónico}

En la tabla 3 se detalla la información del generador de la Unidad 1 de la Central Hidroeléctrica de Salvajina, requerida para realizar de ajuste de la función ANSI 24. 


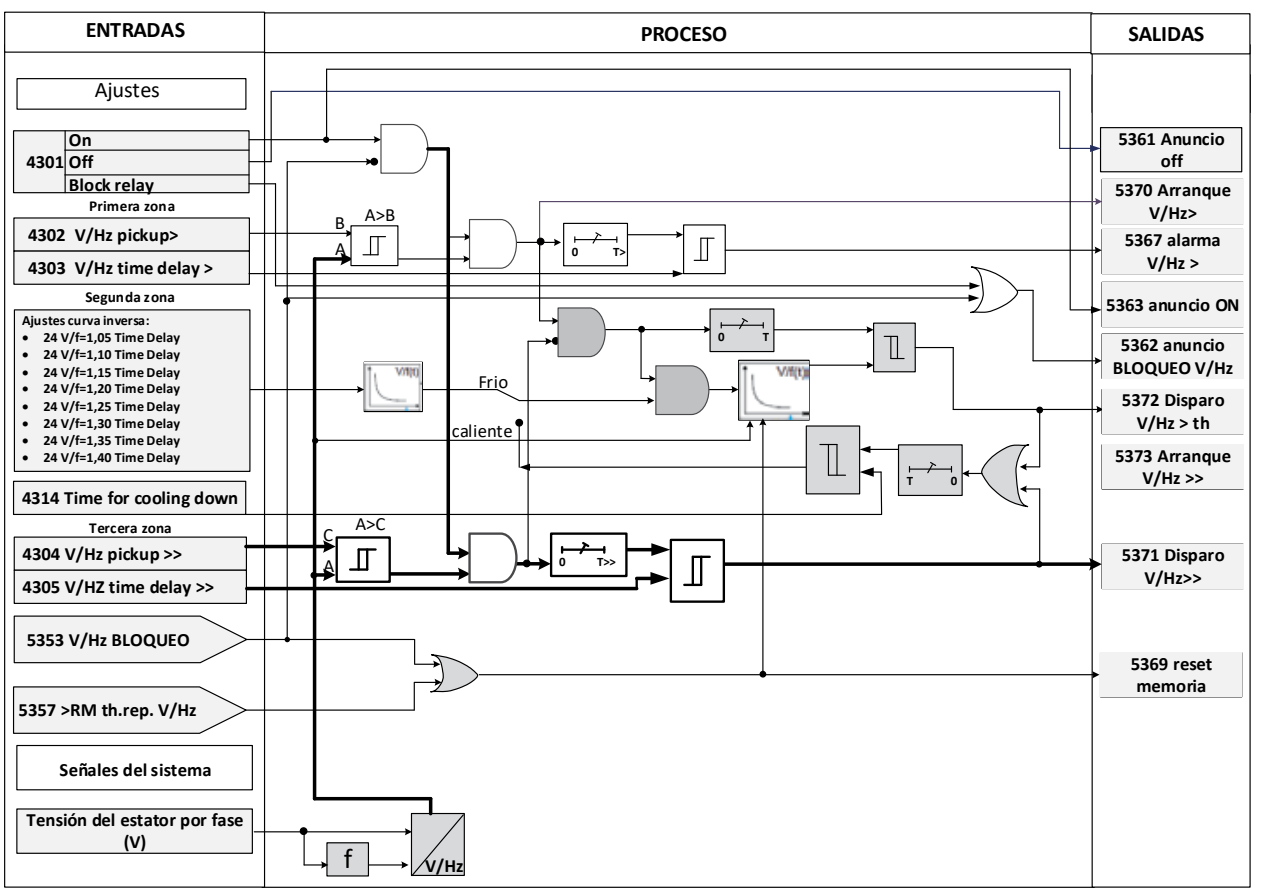

Figura 9. Subdiagrama lógico de decisión de la FDP ANSI 24. Etapa de sobreexcitación por V/Hz>>

Fuente: elaboración propia.

Tabla 3. Parámetros técnicos del generador

\begin{tabular}{|c|c|c|c|c|c|c|c|c|c|c|}
\hline Gen & Vnom(kV) & S (MVA) & FP & $X_{d}$ (p.u.) & $X_{d^{\prime}}$ (p.u.) & $X_{d^{\prime \prime}}$ (p.u.) & $X_{q}$ (p.u.) & $X_{q^{\prime \prime}}$ (p.u.) & $X_{2}$ (p.u.) & $X_{0}$ (p.u.) \\
\hline Salvajina G1 & 13,8 & 125 & 0,94 & 1,16 & 0,35 & 0,26 & 0,83 & 0,3 & 0,28 & 0,2 \\
\hline
\end{tabular}

Fuente: EPSA-Celsia Vicepresidencia de Generación (2018).

A continuación, se muestran los ajustes realizados para la Unidad 1 de la Central Hidroeléctrica de Salvajina:

- [4301] On/Off se selecciona en On.

- [4302] Ajuste de $\frac{U}{f}>$ pickup. Teniendo en cuenta que este escalón corresponde a la señal de alarma se parametriza a un valor de $110 \%$ coordinando con la función de sobretensión, ya que un aumento de la relación $\mathrm{V} / \mathrm{Hz}$ se puede ver con mayor frecuencia por un aumento de la tensión en escenarios de operación en donde se esté suministrando potencia reactiva a la red (Muñoz y Aragón, 2008).

$$
\frac{U}{f}>\text { pick up }=110 \% \frac{U_{\text {nfase }}}{60 \mathrm{~Hz}}=\frac{115 / \sqrt{3}}{60}=\frac{1,217 \mathrm{~V}}{\mathrm{~Hz}}
$$

- [4303] Ajuste $T \frac{U}{f}>$ Time Delay. El rango de selección del relé está entre 0 y 60 segundos y se selecciona 10 segundos, que es ajuste por defecto. Es decir:

$$
T \frac{U}{f}>=10 s
$$

- [4306]- [4313] Ajustes curva característica térmica inversa. Teniendo en cuenta que no se cuenta con la curva $\mathrm{V} / \mathrm{Hz}$ dada por el fabricante del generador, la característica térmica se parametriza con los puntos dados por el manual del relé (SIEMENS, 2009), como se detalla en la tabla 4. 
Tabla 4. Interfaz para el ajuste de los puntos de la característica térmica del grupo generador-transformador

\begin{tabular}{ccc}
\hline \multicolumn{3}{c}{ Característica térmica } \\
\hline No. & Settings & Value \\
\hline 4306 & $\mathrm{U} / \mathrm{f}=1,05$ Time Delay & $6000 \mathrm{~s}$ \\
\hline 4307 & $\mathrm{U} / \mathrm{f}=1,10$ Time Delay & $15 \mathrm{~s}$ \\
\hline 4308 & $\mathrm{U} / \mathrm{f}=1,15$ Time Delay & $10 \mathrm{~s}$ \\
\hline 4309 & $\mathrm{U} / \mathrm{f}=1,20$ Time Delay & $5 \mathrm{~s}$ \\
\hline 4310 & $\mathrm{U} / \mathrm{f}=1,25$ Time Delay & $2 \mathrm{~s}$ \\
\hline 4311 & $\mathrm{U} / \mathrm{f}=1,30$ Time Delay & $1 \mathrm{~s}$ \\
\hline 4312 & $\mathrm{U} / \mathrm{f}=1,35$ Time Delay & $0 \mathrm{~s}$ \\
\hline 4313 & $\mathrm{U} / \mathrm{f}=1,40$ Time Delay & $0 \mathrm{~s}$ \\
\hline
\end{tabular}

Fuente: EPSA-Celsia Vicepresidencia de Generación (2018).

Si se cuenta con las características térmicas V/ $\mathrm{Hz}$-tiempo entregadas por el fabricante del generador y del transformador, se considera como referencia la curva térmica del equipo que para un mismo valor de $\mathrm{V} / \mathrm{Hz}$ se calienta con mayor rapidez. La curva de la FDP ANSI 24 debe estar ajustada por debajo de la curva de referencia, con el fin de lograr su disparo antes de alcanzar el valor límite de calentamiento permitido.

- [4314] Time for cooling down. Tiempo de enfriamiento de la máquina. Su rango de ajuste está entre 0 y $20000 \mathrm{~s}$, se ajusta en el valor defecto de $3600 \mathrm{~s}$.

- [4304] Ajuste de $\frac{U}{f}>>$ pickup. Se propone parametrizar a un valor de $140 \%$ con el fin de prestar un respaldo al escalón 2 de la función de sobretensión ANSI 59.

$$
\frac{U}{f} \gg=140 \% \frac{U_{n f a s e}}{60 \mathrm{~Hz}}=\frac{115 / \sqrt{3}}{60}=\frac{1,549 \mathrm{~V}}{\mathrm{~Hz}}
$$

- [4305] Ajuste de Time Delay T U/f>>. El rango de selección del relé está entre 0 y 60 segundos y se selecciona $0 \mathrm{~s}$, es decir su disparo en esta zona es instantáneo.

\section{RESULTADOS DE LOS AJUSTES DE LA FUNCIÓN ANSI 24 DEL GRUPO GENERADOR-TRANSFORMADOR DE LA UNIDAD 1 DE SALVAJINA}

En la tabla 5 se ilustra la interfaz gráfica de los ajustes de la protección de sobreexcitación cargados al relé 7 UM62. Se recomienda para una mayor precisión en los parámetros de ajuste obtener la característica de calentamiento $\mathrm{V} / \mathrm{Hz}$ real o entregada por el fabricante de los equipos a proteger.

Tabla 5. Interfaz gráfica de ajustes de la protección de sobreexcitación ANSI 24 [5]

\begin{tabular}{lll}
\hline No. & \multicolumn{1}{c}{ Settings } & \multicolumn{1}{c}{ Value } \\
\hline 4301 & Overexcitation protection $(\mathrm{U} / \mathrm{f})$ & On \\
\hline 4302 & $\mathrm{U} / \mathrm{f}>$ Pickup & 1,217 \\
\hline 4303 & $\mathrm{~T} \mathrm{U} / \mathrm{f}>$ Time Delay & 10 \\
\hline 4304 & $\mathrm{U} / \mathrm{f}>>$ Pickup & 1,549 \\
\hline 4305 & $\mathrm{~T} \mathrm{U} / \mathrm{f}>>$ Time Delay & 0 \\
\hline
\end{tabular}

Característica térmica

\begin{tabular}{lll}
\hline No & \multicolumn{1}{c}{ Settings } & \multicolumn{1}{c}{ Value } \\
\hline 4306 & $\mathrm{U} / \mathrm{f}=1.05$ Time Delay & 6000 \\
\hline 4307 & $\mathrm{U} / \mathrm{f}=1.10$ Time Delay & 15 \\
\hline 4308 & $\mathrm{U} / \mathrm{f}=1.15$ Time Delay & 10 \\
\hline 4309 & $\mathrm{U} / \mathrm{f}=1.20$ Time Delay & 5 \\
\hline 4310 & $\mathrm{U} / \mathrm{f}=1.25$ Time Delay & 2 \\
\hline 4311 & $\mathrm{U} / \mathrm{f}=1.30$ Time Delay & 1 \\
\hline 4312 & $\mathrm{U} / \mathrm{f}=1.35$ Time Delay & 0 \\
\hline 4313 & $\mathrm{U} / \mathrm{f}=1.40$ Time Delay & 0 \\
\hline 4314 & Time for Cooling Down & 3600 \\
\hline
\end{tabular}

Fuente: elaboración propia.

\section{CONCLUSIONES}

Se identificaron las consecuencias y los daños que puede provocar la sobreexcitación de un grupo generador-trasformador, por lo cual se evidencia que es muy necesario el ajuste y activación de la función ANSI 24. 
Se presentó de manera clara y ordenada la función ANSI 24 del relé Siemens 7UM62 mediante un diagrama funcional detallado, donde se identificaron las señales de entrada y las señales de salida. Además, se presentó la teoría y la metodología detallada del ajuste de la función ANSI 24 del relé Siemens 7UM62 para un grupo generador-tranformador.

Para realizar un ajuste más preciso, es decir, evitar que el grupo se dañe por sobrecalentamiento debido al incremento de los $\mathrm{V} / \mathrm{Hz}$ o que se dispare antes de su calentamiento, es necesario obtener la característica de calentamiento del equipo a proteger entregada por el fabricante o realizar pruebas para obtener la curva real. Finalmente, se presentó el ajuste de la función ANSI 24 de la Unidad 1 de la Central Hidroeléctrica de Salvajina.

\section{REFERENCIAS}

Ceballos M., J.S.C. (2014). Criterios de ajuste para las protecciones de una unidad de generación de energía hidráulica en una PCH. Caso Amaime. [Trabajo de grado]. Programa de Ingeniería Eléctrica, Facultad de Ingeniería, Escuela de Ingeniería Eléctrica y Electrónica. Universidad del Valle. Cali, Colombia. doi: https://doi.org/10.22201/ fi.25940732e.2012.13n2.020
EPSA-Celsia Vicepresidencia de Generación (2018). Actualización del estudio de coordinación de protecciones U1 ch Salvajina. v1, Cali, Colombia.

IEEE Guide for AC Generator Protection (2006). IEEE Std C37.102-2006 (Revison of IEEE Std C37.102-1995) (pp. 1-177). Recuperado de https://ieeexplore.ieee. org/stamp/stampjsp?arnumber $=4109950$

Muñoz, J.C. y Aragón, L.E. (2008). Guía para la parametrización y pruebas de un relé numérico multifunción de protección de generador. [Trabajo de grado]. Programa de Ingeniería Eléctrica, Facultad de Ingeniería, Departamento de Energética y Mecánica. Santiago de Cali, Colombia. doi: https://doi. org/10.18273/revuin.v18n3-2019013

Muñoz, L. y Caicedo, G. (2018). Protección pérdida de campo de excitación de un generador sincrónico para un relé siemens 7UM62. [Trabajo de grado]. Programa de Ingeniería Eléctrica, Facultad de Ingeniería, Escuela de Ingeniería Eléctrica y Electrónica. Universidad del Valle. Cali. doi: https://doi. org/10.22201/fi.25940732e.2011.12n4.040

Siemens (2009). SIPROTEC Multi-Functional Protective Relay 7UM62. Manual. V4.8;2013. Recuperado de https://www.downloads.siemens.com/download-center/Download.aspx?pos=down-load\&fct=getasset\&id1=DLA06_3361

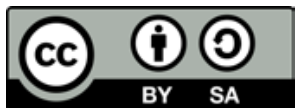

Tecnura • p-ISSN: 0123-921X • e-ISSN: 2248-7638 • Vol. 23 No. $61 \bullet$ Julio - Septiembre de $2019 \bullet$ pp. 57-68 